\title{
Low-power wearable ECG monitoring system for multiple-patient remote monitoring
}

\author{
E. Spanò, S. Di Pascoli, and G. Iannaccone, Fellow, IEEE
}

\begin{abstract}
Many devices and solutions for remote ECG monitoring have been proposed in the literature. These solutions typically have a large marginal cost per added sensor and are not seamlessly integrated with other smart home solutions. Here we propose an ECG remote monitoring system that is dedicated to non-technical users in need of long-term health monitoring in residential environments and is integrated in a broader Internetof-Things (IoT) infrastructure. Our prototype consists of a complete vertical solution with a series of advantages with respect to the state of the art, considering both prototypes with integrated front end and prototypes realized with off-the-shelf components: i) ECG prototype sensors with record-low energy per effective number of quantized levels, ii) an architecture providing low marginal cost per added sensor/user, iii) the possibility of seamless integration with other smart home systems through a single internet-of-things infrastructure.
\end{abstract}

Index Terms - multiple-patient monitoring, ECG, ZigBee, IoT, healthcare.

\section{INTRODUCTION}

$\mathrm{W}$ earable biomedical devices can take advantage of two concurring technology trends. On the one hand, the exponential reduction in cost per function enabled by semiconductor technology, popularized as "Moore's Law", makes low-power and high-performance microcontrollers and radios available at low cost. On the other hand, broadband penetration is very high in large sectors of the population, especially in OECD (Organization for Economic Co-operation and Development) countries. The very same trends are the enabling factors of the so-called "Internet of Things" (IoT), i.e. the deployment of applications based on distributed communicating sensors and actuators.

A market pull is also acting on wearable biomedical devices. Overwhelming demographic trends are expected to increase the demand for healthcare services. By 2050, the world population of age 65 and older will exceed the world population with less than 15 years [1]. By 2030, one in five

Manuscript submitted on October 08, 2014 This work was supported in part by the Italian Ministry of Economic Development through the Cleverhome Grant, by Quantavis s.r.l, by the Tuscany Region under the SED POR-FSE, and by the Italian MIUR and the European Commission through the ENIAC JU grants ERG (contract n. 270722-2) and E2SG (contract $n$. 296131).

E. Spanò, S. Di Pascoli, G. Iannaccone are with the SEED Lab, Polo Universitario Sistemi Logistici and the Dipartimento di Ingegneria dell'Informazione, University of Pisa, 56122 Pisa, Italy (email: elisa.spano@iet.unipi.it, s.dipascoli@iet.unipi.it, g.iannaccone@unipi.it).
US residents will be 65 and older [2]. By 2060, the share of European Union population with 65 years or more will increase from $17 \%$ to $30 \%$ [3].

L. Robert estimated in 2008 [4] that net annual savings in US health care expenditure of $\$ 12$ billion would be achievable with widespread adoption of remote monitoring technologies for chronic diseases. Economic advantages would come through reduced physician and emergency room visits, reduced hospitalization and nursing care at home. About half of the savings would be associated to congestive heart failure patients.

In addition to chronically ill patient, there is a significant portion of healthy population that is both physically active and health conscious, and has been identified as a significant market segment for wearable biomedical products by consumer electronics vendors.

Between these two extremes, there is a large population of overweight, hypertensive, diabetic, elderly people that could benefit from devices and services enabling them to better manage their own health [5].

Many devices and solutions for health monitoring have been proposed in the market and in the technical literature [6] - [25] and [46-49]. They typically consist of monitors of vital signs, wirelessly connected to a smartphone or to a computer that often enable data logging and visualization through a web or mobile application.

Some of the proposed ECG sensor nodes [22-25] are based on a dedicated integrated front end, that sometimes includes a DSP [22-23], and require a second off-the-shelf chip to implement the radio link. However, power consumption mostly in such sensors is mainly due to the radio link and therefore the optimization obtained by the use of the dedicated front-end has a limited impact on the power performance of the complete sensor. In addition, the following sections will show that a general purpose high-performance and highresolution standard ADC can outperform the noise performance of many dedicated front-end chips.

Recent advances in microelectronics and in communication protocols enable cost reduction and performance improvement in these systems, both at the level of individual sensors, and at the level of the infrastructure. Most of all, they can greatly improve usability and reduce the overall cost per patient, therefore fostering market penetration especially in those segments for which health monitoring is more a choice than a necessity.

For these reasons, we here propose an ECG remote 
monitoring system that is dedicated to long-term health monitoring of users/patients in residential environments without assistance, and is integrated in a broader IoT infrastructure. Our proposal has several distinct advantages with respect to the current literature:

- At the infrastructure level, the ECG remote monitoring system can be merged with other biomedical and ambient monitoring systems, because the IoT infrastructure enables the seamless addition of different types of sensors. This issue is discussed in Section II.

- At the local deployment level, the system can monitor multiple patients with the same wireless infrastructure, therefore reducing system cost per patient, perceived complexity, and the marginal cost of adding a monitored patient. We show in Section IV that a single local network can monitor up to six patients per single ZigBee channel at the same time.

- At the individual sensor level, our single ECG sensor has both high signal quality and low power consumption, exhibiting the best figure of merit in terms of Energy per Effective Number of Quantization Levels (EENQL), by more than one order of magnitude with respect to the best results in the literature, which, as discussed in Section V, includes solutions with specifically designed IC frontend.

\section{System Overview}

The architecture of our IoT platform is illustrated in Fig. 1. A detailed description of the platform and of its main components can be found in [26] and [27]. Here, we shall very briefly illustrate the main aspects, with special regard to sensor nodes.

The platform has three main parts: the sensor and actuator networks, the IoT server and the user interfaces for visualization and management.

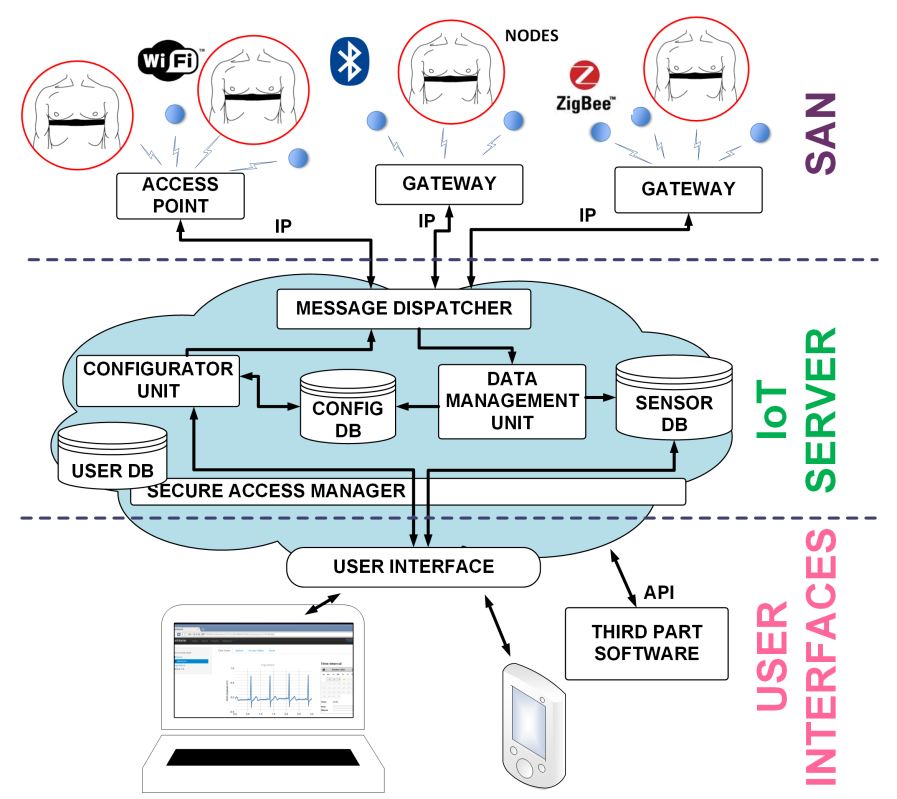

Fig. 1 Block diagram of the Internet of Things platform supporting the inhome Smart Grid
- Sensor and actuator nodes (SANs). Lightweight wearable ECG sensors and other ambient sensors collect data and send them in real time via a wireless protocol (ZigBee, Bluetooth, WiFi) to a gateway connected to the home ADSL router (Fig. 1).

Both the gateway and the message dispatcher are transparent at the logical communication level between sensors and IoT server. The architecture has been developed with the aim of enabling the integration of sensor networks based on different networks protocols (WiFi, ZigBee, Bluetooth...) The only component aware of the local sensor network protocol is the gateway, which runs a firmware that can manage the corresponding protocol. The gateway encapsulates the packets of the sensors in a universal format which preserves all the information present in the native format. Hence sensors send messages in their native format to the IoT server, where the data management unit extracts information and enters it in a "universal" format into the sensor database. When sensors need to be configured or interrogated, the configurator unit prepares a command according to the target sensor protocol.

- The IoT server converts the raw payload from heterogeneous nodes into a "universal" format, containing object identifier, object type, measurement unit, data field, geographical position, and timestamp. Then, it makes the data available to applications and users. In this way, data visualization and processing is separated from measurement and data collection, and does not need to take into account the communication protocol of the originating source. In addition, the IoT server receives data from users in order to configure and manage the SANs.

The main components of the IoT server are illustrated in the cloud of Fig. 1, since they can be part of a distributed information system. The message dispatcher manages the bidirectional communication with the sensor networks, using no information on the network protocol or on the type of application. The data management unit is a collection of software modules interpreting data from sensors and storing them in a universal format in the sensor database. The configurator unit receives inputs from users or applications and translates them into protocol-specific commands to the SANs, consulting the configuration database. Finally, the secure access manager provides access to stored information and SAN configuration only to authorized users and applications, according to information contained in the user database.

- User interfaces. The entire system is configurable and controllable through an intuitive web interface from any computer, smart phone or tablet connected to the internet. In the IoT server, health data can be combined with other data, merged, processed by users and/or authorized clinicians. 
Users with proper access rights can monitor the current sensor status, or query and visualize data in a specific time interval. In Fig. 2, the ECG data visualization thought the web interface is shown.

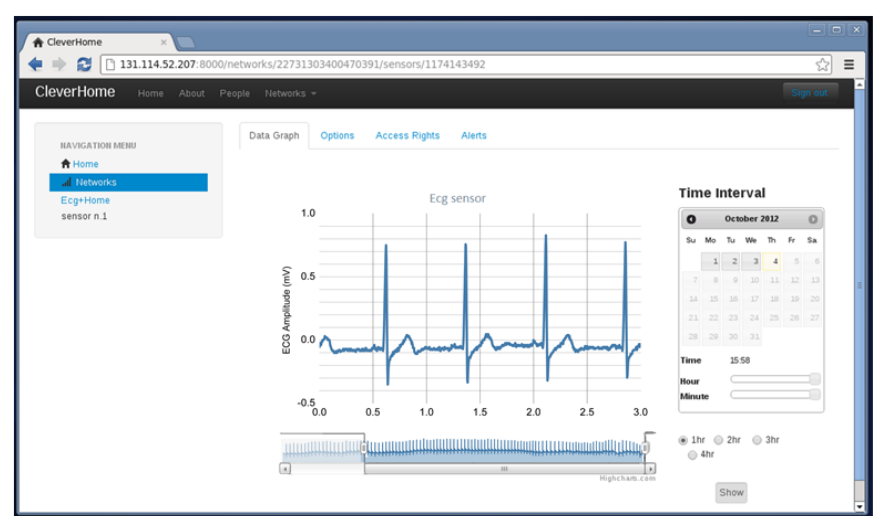

Fig. 2 ECG user interface

\section{ECG SENSOR DESIGN AND IMPLEMENTATION}

The wearable ECG sensor consists of a battery-powered chestbelt enabling the measurement and streaming transmission of electrocardiogram signal during daily routines. The belt has two dry plastic electrodes and the electronic printed circuit board (Fig. 3).

The circuit extracts, filters, amplifies and digitizes the ECG signal, which is then acquired by the microcontroller and wireless sent to the IoT server (Fig. 1). In the first prototype we used the ZigBee communication protocol.
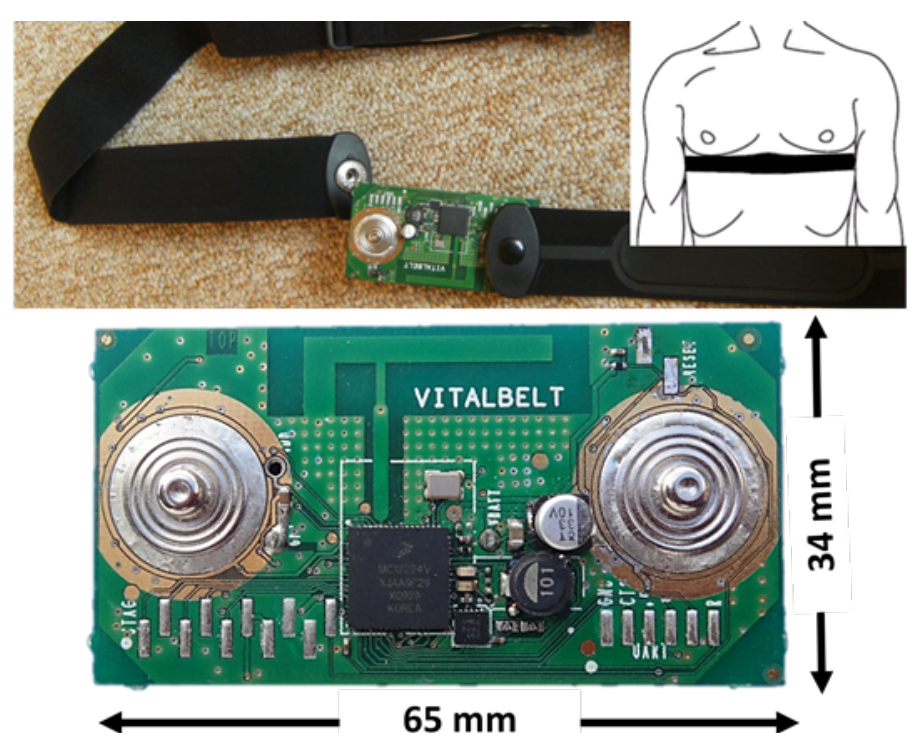

Fig. 3 ECG Belt (top) and ECG board (bottom)

\section{A. ECG signal characteristics and circuit requirements}

An ECG device generally consists of an analog frontend (AFE) circuit and a signal processing circuit. The AFE circuit capabilities and requirements mainly depend on the ECG application, as shown in [28]. Many aspects of the design of an ECG AFE depend on the ECG signal characteristics.

The ECG is a graphical representation of the electric potential difference created on the body surface by heart contraction, which can be detected at different body locations. ECG devices can have a different number of leads, from one to a maximum that is usually 12 , depending on the target application. For monitoring purposes, a one-lead AFE is usually adequate, since only macroscopic behaviors are of interest.

The ECG signal consists of three main components [29]:

1. the actual differential ECG signal;

2. the differential time-varying ECG offset;

3. the common-mode signal.

The actual ECG signal has a bandwidth from $0.05 \mathrm{~Hz}$ to $150 \mathrm{~Hz}$. It has a peak-to-peak amplitude of approximately $1 \mathrm{mV}$ but can reach $3 \mathrm{mV}$.

Motion artifacts (including respiration and body movements) and a poor skin-electrode contact can generate an additional large low frequency offset $( \pm 300 \mathrm{mV})$ that can cause baseline wander. The presence of baseline wander in the ECG signal can affect the interpretation of data, or the computer-based off-line analysis. In the worst case, it can cause the signal chain to saturate.

Finally, a common-mode interference signal can result from coupling to the human body of electromagnetic interference generated by electrical systems, such as AC power lines, electronic devices, fluorescent lights, etc. It is usually the largest noise or disturbance component (up to $1.5 \mathrm{~V}$ ) and needs to be blocked because it can saturate the signal amplifier. This interference must be taken into account only if the ECG sensor is strongly coupled to ground (even a batteryoperated instrument can be coupled to ground by a large ( $\sim 100$ pF) chassis-ground parasitic capacitance).

An instrumentation amplifier with high common-mode rejection ratio (CMRR) is used in the AFE to reduce the common-mode interference. Most systems also typically use a third electrode and a "driven right leg circuit" (DRL) [30], [31], [32] to suppress the interference signal before it can saturate the input amplifier and to further improve the CMRR. A typical single-channel ECG AFE with DRL circuit is shown in Fig. 4. The right leg circuit amplifies the input common mode voltage and feeds it back into the patient's body, in order to suppress the amplitude of the common-mode interference at the amplifier input.

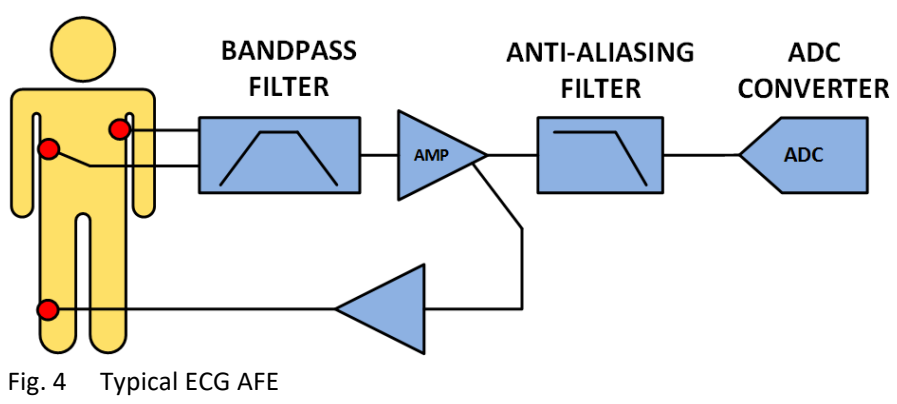


However, few DRL-free ECG systems have been proposed in [33], [34], [35], suggesting that in many situations the active driving of the right leg is not necessary.

We maintain that in our case, and in all similar cases of wearable sensors that are (i) small, (ii) battery operated, (iii) installed close to the body and (iv) differentially connected to the signal source, a DRL can be omitted. Indeed, since the sensor is small and in close proximity to the patient body, the chassis-ground capacitance is negligible with respect to the chassis-human-body capacitance. Hence, the amplitude of the common-mode interference does not threaten to saturate the input amplifier and can be suppressed by the differential amplifier. In this case a simple differential front end with a good CMRR can deliver a medium-high quality signal.

There are strong practical advantages in not using a third electrode: $i$ ) The PCB can be mounted on a chest belt without the need of wired distant electrodes, with improved ease of use ii) the area that picks up magnetic interferences is reduced [35]; iii) the bill of materials and energy consumption are reduced.

\section{B. ECG circuit design}

The design of a battery powered ECG circuit requires a detailed analysis of each subsystem to minimize size, cost and power consumption. Several papers proposed the use of a fully integrated dedicated ECG AFEs, as [22]-[25].

Our system instead, is based on a general-purpose, high performance, high resolution, low power ADC (Texas Instruments ADS1246) [36] that also includes a digital filter. One of the main goals of the former approach is the power reduction that a dedicated ECG integrated front end can provide; however, since the wireless transmission of the ECG signal is the major cause of power consumption in such a system, the total power savings provided are modest at best.

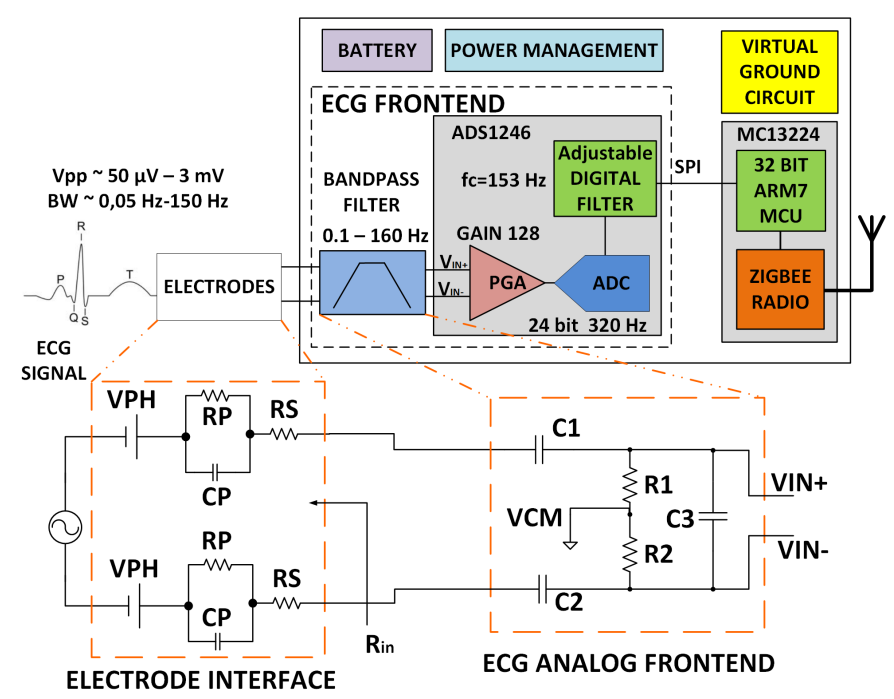

Fig. 5 ECG circuit block diagram and analog circuit schematic. Since the cutoff frequencies of the input filter depend on the electrode impedances, the values indicated in the figure are typical.
Our proposed ECG sensor is designed with the aim of minimizing power consumption for a given signal quality, and non-recurrent (design) costs, and this is best obtained with a general purpose ADC chip.

The cited ADC is preceded by a passive analog frontend and followed by an $\mathrm{MCU}$, which in turn includes the ZigBee radio transceiver (Freescale MC13224 [37]).

A power management block completes the system. The main circuit blocks and the analog frontend schematic are shown in Fig. 5.

The analog front end is a passive two-stage filter connected to conductive rubber electrodes installed into a chest belt (Fig. 3). The first stage is a first-order high-pass filter. Since the spectral content of the baseline wander is usually in the range between 0.05 and $1 \mathrm{~Hz}$ [38], a linear high-pass filtering with a cut-off frequency close to $1 \mathrm{~Hz}$ would be required to reject it. However, most diagnostic devices [39] reduce this frequency down to $0.05 \mathrm{~Hz}$ to avoid ECG information distortion (particularly with respect to the level of the ST segment). In our circuit, we set the cut-off frequency to $0.1 \mathrm{~Hz}$, as a reasonable trade-off between baseline wander rejection and ECG signal distortion reduction.

Unfortunately, such a linear time-invariant filtering cannot completely eliminate the baseline wander that may contain higher frequency components, especially in real-world context (for instance when the patient walks or runs). The residual baseline wander can be suppressed in after the analog to digital conversion using digital algorithms [40], [41], [42].

According to the American Health Association (AHA) specifications, the upper band limit must be higher than $150 \mathrm{~Hz}$ [39]. In our system this cutoff frequency is about $160 \mathrm{~Hz}$, imposed by a first order filter composed by $\mathrm{C}_{3}$ (Fig. 5 ) and the equivalent source resistance $R_{i n}$ at the input of the AFE, dominated by the skin-electrode contact resistance [43]. Experiments with conductive plastic electrodes at $150 \mathrm{~Hz}$, provided $\mathrm{R}_{\text {in }} \cong 500 \mathrm{k} \Omega$. Standard $\mathrm{AgCl}$ electrodes can provide, with accurate placing, a reference impedance of $20 \mathrm{k} \Omega$ [44].

After filtering, the signal is fed into a TI ADS1246 24-Bit analog-to-digital converter[36] powered at $3 \mathrm{~V}$ and sampling at $320 \mathrm{~Hz}$. This chip integrates a low-noise, programmable gain instrumentation amplifier (PGA). Gain selection depends on the amplifier noise, the CMRR, the effective number of bits (ENOB) and the signal dynamic range. For a gain of 128 and $320 \mathrm{samples} / \mathrm{s}$ with a $3 \mathrm{~V}$ power supply it has a nominal input referred noise of only $0.43 \mu \mathrm{V}_{\text {RMS }}\left(0.48 \mu \mathrm{V}_{\mathrm{RMS}}\right.$, measured with a $50 \Omega$ impedance), a maximum input signal before ADC saturation of $23 \mathrm{mV}$, a CMRR higher than $110 \mathrm{dBm}$ and an effective input impedance of $600 \mathrm{M} \Omega$. Under these conditions, the complete ECG front-end has an ENOB of 14, much higher than the ENOB between 8 and 12 of most present-day systems [6], [7], [9], [10]. This ENOB is can be obtained with input impedances up to $70 \mathrm{k} \Omega$, and is only slightly lower with a 500 $\mathrm{k} \Omega$ impedance. Even when compared with fully integrated solutions, [23] and [25], our circuit performance are better in terms of CMRR, input noise and ENOB. It is also worth noting that our noise performance is close to the thermal noise 
limit of the source: assuming a source impedance of $20 \mathrm{k} \Omega$ (see above) and a maximum signal amplitude of $10 \mathrm{mV}$, with a $150 \mathrm{~Hz}$ bandwidth, the maximum achievable ENOB is 15.1 $\mathrm{db}$.

A Texas Instrument Application Note[45] presents a wireless system for ECG recording, based on a similar TI frontend, the three channel ADS1293. Even if this chip is rated for an ENOB of 17 bits, the system described in the application note is not optimized for ECG signals (it lacks a suitable LNA) and hence can extract no more than 12.5 meaningful bits from the source: it has an input noise of about $1.5 \mu \mathrm{V}_{\mathrm{RMS}}$ and an excessively large saturation voltage of 400 $\mathrm{mV}$.

The virtual ground voltage of the ECG differential signal is set to $1.3 \mathrm{~V}$ to shift the common-mode of the signal near to the mid-supply level of the ADC (1.5 V).

After the digital signal conversion, a linear-phase finite impulse response digital filter (FIR) inside the ADS1246 trims the bandwidth to $153 \mathrm{~Hz}$.

The ADS1246 communicates with the signal-processing block of the ECG circuit (Fig. 5) though a standard SPI serial communication interface. The SPI master is a Freescale MC13224 SOC integrating a 32-bit ARM7 microcontroller and the ZigBee radio[37].

The wearable device is powered by a CR2450 $(620 \mathrm{mAh})$ or CR2477 (1000mAh) button battery.

The ECG circuit has been realized as a standard two-layer PCB, Fig. 3. Two button electrodes connect the board to a commercial strap commonly used for heart rate measurement, as shown in Fig. 3. The ECG belt has a printed F-antenna and the output power is set to $0 \mathrm{dBm}$. Receiver sensitivity is about $-96 \mathrm{dBm}$. The board size is $65 \mathrm{~mm} \times 34 \mathrm{~mm} \times 17 \mathrm{~mm}$ and the weight is $16 \mathrm{~g}$ (comprising electrode buttons and battery). The cost of the ECG sensor board, mainly due to the cost of the MC13224 SoC and the ADS1246 analog converter, can be estimated below $\$ 10$ for a production of 1000 pieces.

\section{ECG Data Compression}

To reduce the over-the-air bandwidth in the communication between the ECG node and the gateway, we have introduced a lossless compression of ECG samples before packet formation, with a simple entropy-coding scheme[50].

Since for most of the cardiac cycle the signal is close to zero, small absolute values of the samples have a higher occurring probability than larger ones. This is particularly true for differentially encoded data, which is not affected by offset.

We extracted the probability distribution of ECG data samples and designed an entropy compression scheme, which replaces the 16-bit samples with variable length symbols. Only the most common values (from -150 to 150 ), which have a total probability of more than $99 \%$, are replaced, while the others are transmitted without encoding after a special prefix. This scheme requires only few computing cycles and has a compression ratio of about $50 \%$. Furthermore, tests with ECG signals from common ECG databases[51] showed that the compression ratio is only slightly dependent on the ECG signal quality and statistics.

The compressed stream is divided in blocks of 80 bytes, containing a variable number of compressed samples, and inserted as payload in the ZigBee packets. The reconstructing algorithm is able to resynchronize after every packet and can tolerate missing or defective packets.

\section{ECG Firmware design}

The firmware developed on the ARM7 MCU is responsible for managing the node in a ZigBee network, driving the ADC module, buffering converted samples and sending the information to the gateway using encrypted ZigBee data packets. The flow chart of the program running on the ECG board is shown in Fig. 6 .

The ADS1246 has an internal oscillator and can run while the MCU is in sleep mode. Every $t_{s}=3.125 \mathrm{~ms}\left(\mathrm{f}_{\mathrm{s}}=320 \mathrm{~Hz}\right)$ the ADC DRDY signal goes down indicating that sampling is completed and sending an interrupt to the MCU. The MCU retrieves the 24-bit sample though the SPI extracts the 16 most significant bits and stores them in a circular buffer.

ECG data are transmitted to the receiver as ZigBee packets of near maximum length, in order to minimize packet rate, transceiver duty cycle, and therefore power consumption. The samples are collected into a buffer. When the buffer contains 160 samples a payload of 80 compressed bytes is generated from the buffered samples. The packet rate depends on the compression ratio but is usually close to 4 packets/s. When no compression is used, every packet contains 42 samples corresponding to 84 bytes.

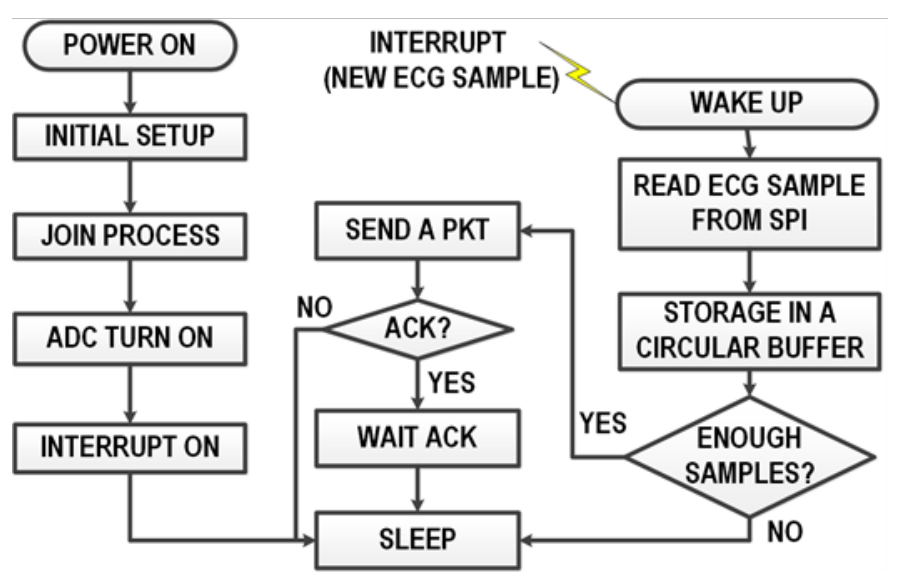

Fig. 6 ECG Board firmware flowchart

To ensure both data confidentiality and authenticity, each packet is encrypted by the ZigBee stack with a 128-bit key and uses the 32-bit MIC (Message Integrity Code) created using the 128-AES algorithm [52], as required by the ZigBee specification [53].

The ECG board is programmed as an End Device ZigBee node. The ZigBee protocol automatically manages to switch the radio off between transmissions and to let the go in sleep during idle. 


\section{EXPERIMENTAL RESULTS}

An actual deployment of the system was used to evaluate key aspects of the system architecture, in particular the possibility to monitor the ECG signal of multiple patients in a large area and for a long time and the possibility to access ECG data through the web interface.

The test deployment consisted of six ECG sensor nodes, a ZigBee gateway and an IoT server [26], [27]. The IoT server was installed on a Linux machine. The ZigBee gateway has been configured to connect to the server, through an ADSL router.

Various tests have been performed in order to evaluate:

A. quality of the ECG signal;

B. maximum range;

C. maximum data rate in terms of maximum number of ECG belts that can be connected to a single gateway at the same time;

D. power consumption.

\section{A. ECG signal quality}

Fig. 7 shows the raw measurement data from the ECG sensors recorded from a healthy 35-year-old man. As illustrated in Fig. 7, the quality of the wireless ECG signal is very good and allows to clearly identify the QRS complex, the $\mathrm{P}$ and $\mathrm{T}$ waves and the ST segment.

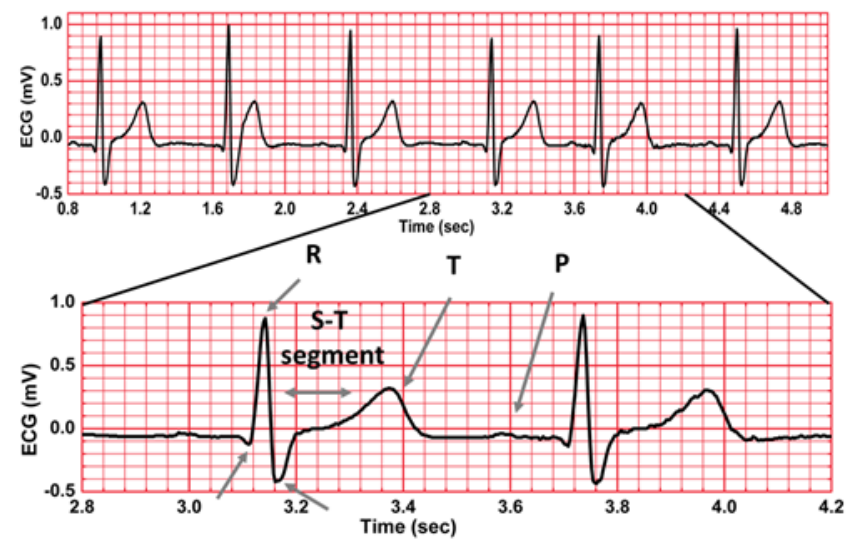

Fig. 7 ECG signal waveform

As expected due to the cutoff high pass frequency (III.B, a baseline drift can be observed due to respiration and patient movements (Fig. 8).

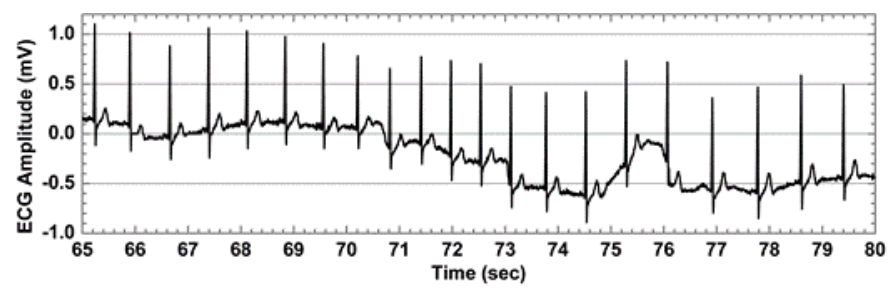

Fig. 8 ECG baseline drift due to respiration and movements

\section{B. Maximum range test}

Maximum range tests has been performed both outdoor than indoor confirming the ZigBee range of $30 \mathrm{~m}$ indoor and more than $70 \mathrm{~m}$ outdoor.
The outdoor open space range measurement has been performed in an empty parking area, with a low probability of multipath reflections from buildings. Both the node and the gateway have been mounted on a plastic pole at a height of $1.5 \mathrm{~m}$ from the ground. The average power at the receiver as a function of distance is shown in Fig. 9. The effect of ground reflection is clearly visible.

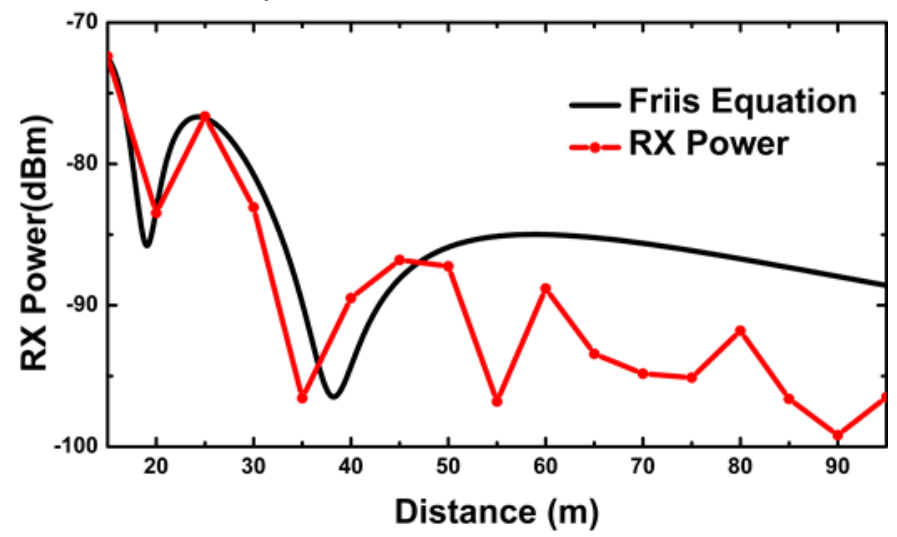

Fig. 9 Comparison between measured RX power and Friis equation including ground reflection (considering nominal system parameters and a asphalt relative permittivity of 18 )

\section{Data loss and maximum number of nodes}

In our system, each ECG node communicates to the ZigBee/Ethernet Gateway over a single hop or multi-hop ZigBee communication. The number of nodes that a ZigBee router or a gateway can support depends on the data transmission rate of each node, the usage of the ZigBee APS acknowledgement (ACK) mechanism and the gateway bandwidth. To measure the packet data loss in multiuser ECG monitoring and determine the maximum number of ECG belts that can be reliably connected to a single gateway, we deployed a star network without routers. In this configuration, each node sends data directly to the gateway, which represents the system bottleneck.

Four test configurations have been performed: with and without data compression, with and without ACK. All the tests were performed using a single ZigBee channel.

For test using the ACK mechanism, due to the high data rate (in comparison to other ZigBee sensors), the maximum number of retries of the APS ACK has been set to 1 (instead of 3 ) for a total of 2 tries and the ACK wait duration has been set to $200 \mathrm{~ms}$ (set by default to $1.8 \mathrm{~s}$ ). During each test, several 10-minute acquisitions have been performed.

In Fig. 10, the average packet loss as a function of the number of concurrent ECG devices is shown for each test configuration (corresponding to more than 8000 packets per node without compression and more than 4000 packets per node with compression). The average packet loss is the ratio of the number of packets not successfully delivered to the number of messages generated by all ECG devices.

Fig. 10 shows that the acknowledge mechanism actually increases data loss for the ECG sensor (it could be useful for lower data rate sensors), while compression strongly increases the number of ECG devices that can be simultaneously used for a given packet loss rate. 


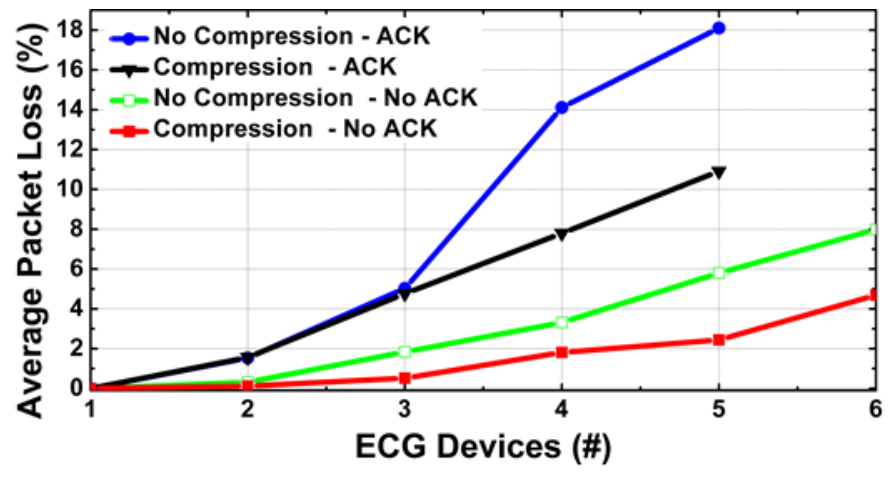

Fig. 10 :ECG Average packet loss as a function of the number of users.

If we assume, as in [6], that a 5\% data loss does not compromise the clinical usefulness of ECG signals, we can estimate in 5 the maximum ECG boards that a single ZigBee coordinator can support. The corresponding total raw data rate is about $20 \mathrm{kbit} / \mathrm{s}$, well below of the rated capacity of a single ZigBee channel (250 kbit/s).

\section{ECG battery lifetime}

To estimate the suitability of the ECG belt as a long-term wearable sensor, we have evaluated energy consumption. Current draw from the power supply varies during active, transmitting and sleep mode of the device; therefore we have performed the measurement with an oscilloscope.

During the test, the ECG board is powered by a tabletop DC power supply with $3 \mathrm{~V}$. The current consumption is measured with an oscilloscope probe over a $1 \Omega$ resistor in series with the power supply. The plot in Fig. 11 shows the current consumption during data transmission, ECG sampling and MC13224 sleep.

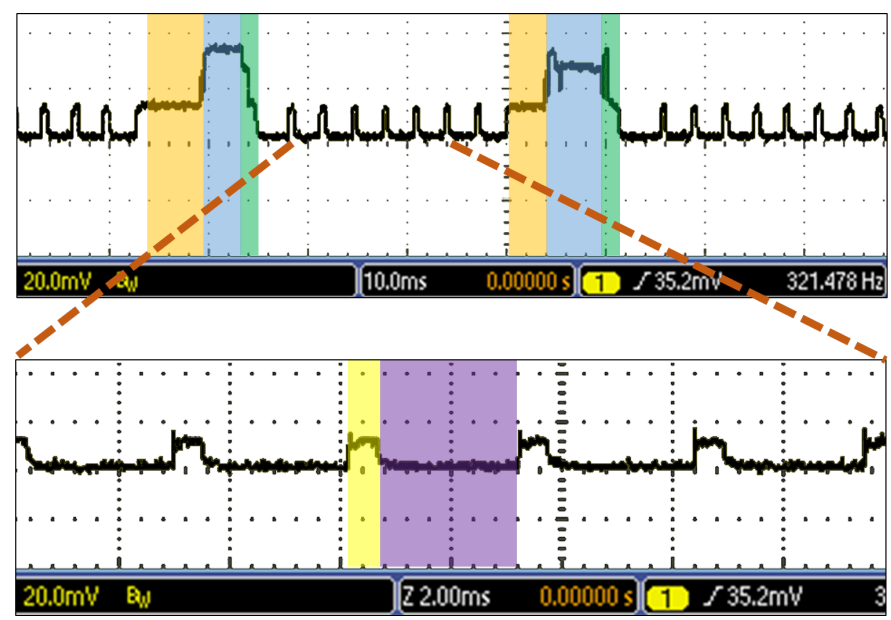

Fig. 11 Power consumption during data transmission and acknowledgement

Six intervals can be identified during the transmission of a packet without ACK and two during ECG sampling. For each interval, power consumption and duration are measured.

The radio link has an average current consumption of $0.95 \mathrm{~mA}$ with compression and of $1.77 \mathrm{~mA}$ without. The sampling and processing of the samples, at 320 samples/s, uses another $3.12 \mathrm{~mA}$. The resulting battery lifetime in continuous operation using a lithium $3 \mathrm{~V}$ battery with a $650 \mathrm{mAh}$ capacity is then 160 hours ( $>6$ days), with compression and 132.71 hours ( $>5$ days) without.

This resulting battery lifetime is comparable to that of the sensor proposed in [7], where data are sampled at $250 \mathrm{~Hz}$ and stored into a micro SD card.

Considering other wireless systems, the obtained battery life is very good. Other proposed solutions with point-to-point communication are only able to ensure a battery life of about 28 hour sampling at $300 \mathrm{~Hz}$ and 8-bit of resolution and transmitting them through ZigBee [9], or a lifetime of 33 hours sending ECG data through Bluetooth [10]. This is the combined result of using a low-energy protocol such as ZigBee, of minimizing transmission time, and of careful design of the AFE.

\section{COMPARISON WITH RELATED WORKS}

In this section we compare our results with related works. Given the multiplicity of aspects addressed in our work, and the fact that we have developed a complete vertical system, from the ECG sensors to the IoT architecture, we prefer to divide our comparison in two parts. First, we perform a comparison at the level of the single ECG boards, showing that our prototype has an inherent strength in terms of energy consumption and signal quality, synthesized in a single figure of merit (energy per effective number of quantized levels). Then, we perform a comparison at the level of complete system and architecture, focusing on the communication aspects and on the integration with other sensors and applications.

\section{A. ECG Boards}

The proposed ECG sensor is designed to meet the AHA specifications [39], as summarized in Table 1.

In order to make a comparison with related works on ECG boards, we have selected the prototypes presented in the literature that satisfy the following set of requirements:

- Battery-powered, long term operation ( $>=24$ hours) and wearable;

- Good quality signal (CMRR $>=60 \mathrm{dBm}$, at least 10 bits, sampling frequency of at least $100 \mathrm{~Hz}$ );

- Complete sensors (AFE, ADC, MCU, Power Management, Radio or storage unit).

TABLE 1: PERFORMANCE SUMMARY OF OUR ECG WEARABLE SENSOR

\begin{tabular}{|l|l|l|}
\hline QUANTITY & AHA SPECIFICATIONS & ECG wearable sensor \\
\hline ADC ENOB & $>8-10 \mathrm{bit}$ & $14 \mathrm{bit}$ \\
\hline ADC sample rate & $>120 \mathrm{~Hz}$ & $320 \mathrm{~Hz}$ \\
\hline Leads & From 1 to 12 & 1 \\
\hline Input noise & $<20 \mu \mathrm{V}_{\mathrm{RMS}}$ & $0.48 \mu \mathrm{V}_{\mathrm{RMS}}$ \\
\hline CMRR & $>60 \mathrm{dBm}$ & $>112 \mathrm{~dB}$ \\
\hline $\mathbf{f}$ min & $0.05-0.5 \mathrm{~Hz}$ & $0.1 \mathrm{~Hz}$ \\
\hline f max & $40-100 \mathrm{KHz}$ & $153 \mathrm{~Hz}$ \\
\hline Input range & $20 \mu \mathrm{V}-3 \mathrm{mV}$ & $20 \mu \mathrm{V}-20 \mathrm{mV}$ \\
\hline Lifetime & $>1-2$ days & $>5$ days (compression) \\
& & $>6$ days (no compression) \\
\hline
\end{tabular}


TABle 2 Performance Comparison of Wearable ECG Wireless Sensors for Long Term Monitoring

\begin{tabular}{|c|c|c|c|c|c|c|c|c|c|c|c|}
\hline & {$[6]$} & [7] & {$[10]$} & [11] & [21] & $\begin{array}{l}{[22]-} \\
{[23]}\end{array}$ & [45] & {$[46]$} & {$[47][48]$} & [49] & $\begin{array}{c}\text { This } \\
\text { work }\end{array}$ \\
\hline ADC (bits) & 10 & 12 & 12 & 10 & 10 & 12 & $\begin{array}{c}24(12.5 \\
\text { ENOB, } 3 \\
\text { channels) }\end{array}$ & 14 & 10 & 10 & $\begin{array}{l}16.5(14 \\
\text { ENOB) }\end{array}$ \\
\hline fs $(\mathrm{Hz})$ & 200 & 250 & 512 & 500 & 400 & 256 & 160 & 100 & 125 & 500 & 320 \\
\hline $\mathbf{f}_{\mathrm{L}}(\mathrm{Hz})$ & $\mathrm{n} / \mathrm{a}$ & 0.5 & 0.05 & 0.5 & $\mathrm{n} / \mathrm{a}$ & $\mathrm{n} / \mathrm{a}$ & $\mathrm{n} / \mathrm{a}$ & 0.8 & 0.15 & 1 & 0.1 \\
\hline $\mathbf{f}_{\mathrm{H}}(\mathrm{Hz})$ & $\mathrm{n} / \mathrm{a}$ & 125 & 150 & 85 & $\mathrm{n} / \mathrm{a}$ & $\mathrm{n} / \mathrm{a}$ & $\mathrm{n} / \mathrm{a}$ & $\mathrm{n} / \mathrm{a}$ & $250(\mathrm{sic})$ & 150 & 153 \\
\hline CMRR (dB) & 60 & 99 & $\mathrm{n} / \mathrm{a}$ & 60 & $\mathrm{n} / \mathrm{a}$ & $>100$ & $\mathrm{n} / \mathrm{a}$ & $\mathrm{n} / \mathrm{a}$ & $\mathrm{n} / \mathrm{a}$ & 50 & $>112$ \\
\hline $\begin{array}{l}\text { Input noise } \\
\text { (nVRMS) }\end{array}$ & $\mathrm{n} / \mathrm{a}$ & 3600 & $\mathrm{n} / \mathrm{a}$ & $\begin{array}{l}1000 \\
\left(\mathrm{~V}_{\mathrm{pp}}\right)\end{array}$ & $\mathrm{n} / \mathrm{a}$ & $<1000$ & 1500 & $\mathrm{n} / \mathrm{a}$ & $\mathrm{n} / \mathrm{a}$ & $\mathrm{n} / \mathrm{a}$ & 48 \\
\hline DLR circuit & $\mathrm{n} / \mathrm{a}$ & YES & YES & YES & NO & NO & YES & $\mathrm{n} / \mathrm{a}$ & NO & YES & NO \\
\hline $\begin{array}{l}\text { Commun. } \\
\text { protocol }\end{array}$ & $\begin{array}{l}\text { Simpl } \\
\text { iciTI }\end{array}$ & $\begin{array}{c}\text { No } \\
\text { transm } \\
\text { ission }\end{array}$ & $\mathrm{BT}$ & ANT & $\begin{array}{c}\text { Propriet } \\
\text { ary }+ \\
\text { BT }\end{array}$ & BLE & BLE & $\begin{array}{c}\text { Modified } \\
\text { ZigBee }\end{array}$ & BT & BT & ZigBee Pro \\
\hline $\begin{array}{l}\text { Indoor Link } \\
\text { (m) }\end{array}$ & $\sim 30$ & 0 & $\sim 10$ & $\sim 30$ & 10 & $\sim 10-20$ & $\mathrm{n} / \mathrm{a}$ & 30 & $\mathrm{n} / \mathrm{a}$ & 65 & $\sim 30$ \\
\hline Packet Loss & $<5 \%$ & - & $\mathrm{n} / \mathrm{a}$ & $\mathrm{n} / \mathrm{a}$ & $\mathrm{n} / \mathrm{a}$ & $\mathrm{n} / \mathrm{a}$ & $\mathrm{n} / \mathrm{a}$ & $\begin{array}{l}10 \%(5 \\
\text { nodes }) \\
4 \%(1 \\
\text { node })\end{array}$ & $\mathrm{n} / \mathrm{a}$ & $\mathrm{n} / \mathrm{a}$ & $\begin{array}{c}<0,025 \%- \\
1 \text { node } \\
<6 \%-6 \\
\text { nodes }\end{array}$ \\
\hline Voltage (V) & 9 & 3.7 & 3.7 & 3 & $\begin{array}{c}1.4- \\
\text { sensor }\end{array}$ & 3.7 & 3.6 & 4.1 & 3.3 & 3.7 & 3 \\
\hline I Avg. (mA) & 4 & 2.72 & 31 & 17 & $\mathrm{n} / \mathrm{a}$ & 5.8 & 15 & 41.8 & 0.5 & 20 & 4.07 \\
\hline Power $(\mathrm{mW})$ & 36 & 10 & 115 & 51 & $\begin{array}{c}1.5+ \\
\text { FPGA + } \\
\text { BT }\end{array}$ & 21.48 & 54 & 171.4 & 1.65 & 74 & 12 \\
\hline $\begin{array}{l}\text { Battery } \\
\text { (mAh) }\end{array}$ & 180 & 600 & 1100 & 256 & $\mathrm{n} / \mathrm{a}$ & 400 & 720 & 500 & 180 & 280 & 650 \\
\hline Life (h) & 45 & 220 & 33 & 15 & $\mathrm{n} / \mathrm{a}$ & 70 & $\mathrm{n} / \mathrm{a}$ & 10 & 360 & 24 & 160 \\
\hline EENQL (nJ) & 176 & $\begin{array}{l}10(\text { no } \\
\text { TX) }\end{array}$ & 59 & 100 & $\begin{array}{c}>>4(4+ \\
\text { BT }+ \\
\text { FPGA })\end{array}$ & 41 & $\begin{array}{l}19 \text { (per } \\
\text { channel) }\end{array}$ & 104.6 & 12.9 & 144.6 & 2 \\
\hline
\end{tabular}

These criteria allow us to select among the many recent proposals of portable ECG boards in the literature [6], [7], [10], [11], [21] , [22]-[23] [45-49].

The comparison is shown in Table 2.

Our system does not use driven right leg circuit (while comparison prototypes except [21][22] [47-48] do), which is not needed, as we have discussed in section III. The absence of the third electrode makes the proposed system more compact, more easily wearable, and more energy efficient.

Even if Ref. [21] and in Ref. [22] and [23] propose low power fully integrated $\mathrm{AFE}$ and $\mathrm{ADC}$, reducing acquisition power consumption, the reduction is negligible when summed to the ECG signal streaming wireless transmission. Moreover the proposed integrated solutions employ ADCs with a lower resolution (ENOB) than our sensor.

Packet loss comparison is possible only with Refs. [6] and [46], for which data are available. With one sensor per gateway, Ref. [6] obtains a packet loss smaller than 5\%. We obtain a packet loss lower than $0.025 \%$ with one sensor per gateway, and of $5 \%$ with 6 sensors connected to the same gateway. Ref. [46] has a minimum packet loss of about $4 \%$ (even with 1 node). We should mention that we do not know if the measurement conditions are the same.

A further advantage with respect to Ref. [6] is that we use a ZigBee protocol that allows multiple sensors per gateway, whereas they use a dedicated access point per each ECG board. This means a much lower marginal cost of each sensor in our case.

Our system has also an advantage in terms of the used network protocol: ZigBee has specific Application Profiles for Home automation [54], and the ECG sensors can be much more easily integrated in home automation systems. On the other hand other ECG sensors use SimpliciTI [6], Bluetooth [10], [21], ANT [11], and Bluetooth Low Energy [22], [23] and [45] which are simply used for two-way links. 
Except for Ref. [7], where data are not transmitted but stored in a local SD card, our solution has a battery lifetime far larger than other similar proposals.

We propose to use the energy per effective number of quantization levels (EENQL) as a relevant figure of merit for sensors [55]. In doing so, we extend the concept of an established figure of merit for AD converters [56].

At a high level of abstraction, we can consider the whole sensor system as a single $\mathrm{AD}$ converter, including in the definition also the AFE, the processing in the microcontroller, and the radio link.

The definition we use is

$$
\mathrm{EENQL}=\frac{\mathrm{P}_{\mathrm{B}}}{f_{S} 2^{\mathrm{ENOB}}}
$$

Where $\mathrm{P}_{\mathrm{B}}$ is the total power consumption of the battery-powered parts of the system (in our case, only the ECG board). We include the ENOB of the complete sensor instead of the raw number of bits because the former takes into account the whole sensor performances. In our case, we have been able to measure the ENOB of the whole system. In the case of comparison circuits, we do not have the ENOB, and we have largely overestimated it using the nominal number of bits indicated.

Nevertheless, one can see in the last row of Table 2 that our ECG board exhibits an EENQL lower by more than one order of magnitude compared to solutions with wireless transmission, and by a factor 5 compared to the local storage solution. Insufficient information is available on [21], because the power consumption of the Bluetooth module and of the controlling and classifying FPGA is not provided. Even excluding those components (which instead are likely to be dominant), the EENQL would be twice our case.

Again, there is no single aspect responsible for this result. The high number of bits of the ADC, the low noise AFE, and the absence of DRL have a role, as well as the very low duty cycle of the transmitter, due to the data compression and to the use of maximum length packets.

\section{B. Health monitoring systems}

The growing research interest in remote health and patient monitoring has resulted in a multiplicity of proposed systems, many of which focusing on ECG monitoring.

In this section we compare our system with other ECG monitoring solution in residential environments. Comparison among complete health monitoring systems is more difficult, because there are many proposed systems with different scope and different components.

We choose to compare our system with solutions that include ECG monitoring with wireless systems in residential settings, and satisfy a "minimum distance" criterion with respect to our work. Six systems make the cut, listed in Table 3.

Let us also point out that there are no common or apparent performance metrics, so that the comparison is often qualitative. The following aspects differentiate our system:
The first difference is in terms of connection to the internet: The gateway is a PC in [12], [13], and [14], an Android handheld device in [16], a MSP430-based board in [6], and a Cortex M3/4-based board in our case. Our system and that of [6] put the least hardware requirements on the gateway. In addition, in our case the gateway collects data from multiple sensors and encapsulates raw sensor data in a secure TCP/IP packet, whereas in [6] there is one gateway per sensor. Therefore, our system has the lowest total cost of gateways for a given number of sensors, and hence the lower marginal cost of adding one ECG sensor to the system. [46] Concentrates on the routing protocol and does not provide details on the gateway hardware.

\section{- Integration with other smart home systems}

One of the main factors that make difficult a real in-home deployment of a health monitoring systems is the integration of different communication protocols in the same platform and the possibility to combine and integrate health sensors with smart home sensors. Among comparison systems, only [13] and [14] address such integration.

The solution proposed in [13] includes a distributed application over the application layer of each wireless sensor network stack connected to the system. This solution is lacking in flexibility requiring substantial modifications of existing network and nodes before they can be added to the system.

Our proposed system integrates different sensor communication protocols at the level of the communication layer, encapsulating all protocoldependent information in TCP/IP packets that are interpreted only at the ends (in the gateway and in the IoT server). This enables concurrent applications to share connectivity and possibly hardware resources without the need of modifying each sensor.

As the solution presented in [14], our system integrates in the same monitoring platform ambient and wearable sensors and can wirelessly cover a whole building. The main difference with respect to our architecture is that [14] uses a local PC to collect and store data, whereas our architecture is based on a distributed remote server, providing the advantages typical of cloud-based systems.

\section{- Secure and differentiated access to data}

This is specifically treated only in our proposal, and in none of the comparison systems.

\section{- Internet connection and cost of gateways}


TABLE 3: COMPARISON AMONG THE PROPOSED SYSTEM AND OTHER RECENTLY PROPOSED HEALTH MONITORING SYSTEMS

\begin{tabular}{|l|l|l|l|l|c|}
\hline & $\begin{array}{l}\text { Health } \\
\text { others }\end{array}$ & Protocol & $\begin{array}{l}\text { Indoor } \\
\text { coverage }\end{array}$ & $\begin{array}{l}\text { Data } \\
\text { storage }\end{array}$ & $\begin{array}{l}\text { Gateway } \\
\text { Hardware }\end{array}$ \\
\hline$[\mathbf{1 4}]$ & YES & 802.15 .4 & house & local & - \\
\hline$[\mathbf{1 5}]$ & NO & ZigBee & $30 \mathrm{~m}$ 1-hop & local & - \\
\hline$[\mathbf{6}]$ & NO & SimpliciTI & $30 \mathrm{~m}$ 1-hop & remote & MSP430 MCU \\
\hline$[\mathbf{1 2}]$ & NO & BT & $10 \mathrm{~m}(\mathrm{BT})$ & remote & atom PC \\
\hline$[\mathbf{1 3}]$ & YES & Multiple & n/a & remote & PC \\
\hline$[\mathbf{1 6}]$ & NO & Multiple & n/a & remote & Android device \\
\hline$[\mathbf{2 1}]$ & NO & Prop + BT & $10 \mathrm{~m}(\mathrm{BT})$ & remote & Smartphone \\
\hline$[\mathbf{4 6}]$ & YES & ZigBee & $30 \mathrm{~m}$ & remote & n/a \\
\hline This & YES & Multiple & house & remote & $\begin{array}{c}\text { CortexM3 } \\
\text { work }\end{array}$ \\
\hline & & & & & MCU \\
\hline
\end{tabular}

\section{CONCLUSION AND FUtURE WORK}

We have proposed a wireless wearable ECG monitoring system embedded in an IoT platform that integrates heterogeneous nodes and applications, has a long battery life, and provides a high-quality ECG signal. The system allows monitoring multiple patients on a relatively large indoor area (home, building, nursing home, etc.).

Our ECG sensor exhibits the record-low EEQNL figure of merit (energy per effective number of quantized levels) of all solutions with both discrete and integrated frontends available in the literature. As we have shown, this result is obtained through a careful set of choices at the level of components, circuit solutions, and algorithms.

We would like to stress the fact that a dedicated front-end chip is not enough to achieve an advantage in terms of overall sensor performance. The latter depends on the optimization of the whole sensor, that is more practically done using off-theshelf components. Indeed, our proposed ECG sensor, based on a high performance $\mathrm{ADC}$ and a microprocessor-radio combo chip, provides much better performance, in terms of power consumption and noise, than many proposed systems based on a purposely designed front end chip [22-25].

Another remarkable feature of our system is a very low marginal cost per added sensor, since our architecture enables a single low-cost gateway to manage multiple sensors.

Future work will focus on monitoring additional healthrelated parameters using a broader combination of transducers, sensors, and correlation techniques, and on improving system reliability and robustness to patient movement and connectivity losses.

\section{REFERENCES}

[1] A.E. Raftery, N. Li, H. Ševčíková, P. Gerland, G.K. Heilig, "Bayesian probabilistic population projections for all countries," in Proc. 2012 National Academy of Sciences, vol. 109 p. 13915-139121.

[2] G. Vincent, V. Velkoff, "The Next Four Decades: The Older Population in the United States 2010 to 2050", Washington, DC: US Census Bureau, US Government Printing Office, 2010.
[3] European Commission, "The 2012 Ageing Report: Economic and budgetary projections for the 27 EU Member States (2010-2060)," 2012.

[4] L. Robert, "Vital Signs via Broadband: Remote Monitoring Technologies Transmit Savings," Better Health Care Together Coalition, p. 1, Oct. 2008.

[5] IBM Institute for Business Value, "The future of connected health devices", Executive Report, 2011.

[6] R. S. Dilmaghani, H. Bobarshad, M. Ghavami, S. Choobkar, C. Wolfe, "Wireless sensor networks for monitoring physiological signals of multiple patients.," IEEE transactions on biomedical circuits and systems, vol. 5, no. 4, pp. 347-56, Aug. 2011.

[7] M. K. Delano and C. G. Sodini, "A long-term wearable electrocardiogram measurement system," in 2013 IEEE International Conference on Body Sensor Networks, 2013, pp. 1-6.

[8] E. S. Winokur, M. K. Delano, and C. G. Sodini, "A wearable cardiac monitor for long-term data acquisition and analysis," IEEE Trans. Biomed. Eng., vol. 60, no. 1, pp. 189-92, Jan. 2013.

[9] N.J. Kim, J.H. Hong, T.S. Lee, "A Study on Power Consumption and Transmission Rate in ECG Signal Processing in Mobile Environment," IFMBE Proceedings, Vol. 14, 2007, pp.4107-4110.

[10] I.-J. Wang, L.-D. Liao, Y.-T. Wang, C.-Y. Chen, B.-S. Lin, S.-W. Lu, C.-T. Lin, "A Wearable Mobile Electrocardiogram Measurement Device with Novel Dry Polymer-based Electrodes," in Proc. 2010 IEEE Region 10 Conference, Fukuoka, Japan, 21-24 Nov. 2010, pp. 379-384.

[11] E. Nemati, M. Deen, and T. Mondal, "A wireless wearable ECG sensor for long-term applications," IEEE Communications Magazine, vol. 50, no. 1, pp. 36-43, Jan. 2012.

[12] L. Fanucci, S. Saponara, T. Bacchillone, M. Donati, P. Barba, I. Sanchez-Tato, and C. Carmona, "Sensing Devices and Sensor Signal Processing for Remote Monitoring of Vital Signs in CHF Patients," IEEE Trans. Instr. and Meas. vol. 62, no. 3, pp. 553-569, Mar. 2013.

[13] J. M. Corchado, J. Bajo, D. I. Tapia, and A. Abraham, "Using heterogeneous wireless sensor networks in a telemonitoring system for healthcare," IEEE transactions on information technology in biomedicine, vol. 14, no. 2, pp. 234-40, Mar. 2010.

[14] S.-J. Jung, T.-H. Kwon, and W.-Y. Chung, "A new approach to design ambient sensor network for real time healthcare monitoring system," IEEE Sensors Journal, pp. 576-580, 2009.

[15] K. Malhi, S.C. Mukhopadhyay, J. Schnepper, M. Haefke, H. Ewald, "A ZigBee-Based Wearable Physiological Parameters Monitoring System," IEEE Sensors Journal, vol. 12, no. 3, pp. 423-430, Mar. 2012.

[16] W.-T. Sung, J.-H. Chen, and K.-W. Chang, "Mobile Physiological Measurement Platform With Cloud and Analysis Functions Implemented via IPSO," IEEE Sensors Journal, vol. 14, no. 1, pp. 111123, Jan. 2014.

[17] J. Gomez-Clapers and R. Casanella, "A Fast and Easy-to-Use ECG Acquisition and Heart Rate Monitoring System Using a Wireless Steering Wheel," IEEE Sensors Journal, vol. 12, no. 3, pp. 610-616, Mar. 2012.

[18] Y. Chuo, M. Marzencki, B. Hung, C. Jaggernauth, K. Tavakolian, P. Lin and B. Kaminska, "Mechanically flexible wireless multisensor platform forhuman physical activity and vitals monitoring", IEEE trans. biomed. circuits and systems., vol. 4, no. 5, pp.281 -294, 2010.

[19] L. Yan, H.-J. Yoo, "A Low-Power Portable ECG Touch Sensor with Two Dry Metal Contact Electrodes," JSTS: Journal of Semiconductor Technology and Science, vol. 10, no. 4, pp. 300-308, Dec. 2010.

[20] X. Zhang, H. Jiang, L. Zhang, C. Zhang, Z. Wang, X. Chen, "An energy-efficient ASIC for wireless body sensor networks in medical applications," IEEE transactions on biomedical circuits and systems, vol. 3, no. 1, pp. 11-18, Feb. 2010.

[21] S. Lee, J. Hong, K. Lin, C.H. Hsieh, M. Liang, S.Y. Chien, "Low-Power Wireless ECG Acquisition and Classification System for Body Sensor Networks," IEEE Journal of Biomedical and Health Informatics, vol. 19, no. 1 pp. 236-246, Jan. 2015.

[22] M. Altini, S. Polito, J. Penders, H. Kim, N. van Helleputte, S. Kim, F. Yazicioglu, "An ECG Patch Combining a Customized Ultra-Low-Power ECG SoC with Bluetooth Low Energy for Long Term Ambulatory Monitoring," in Proceedings of the 2nd Conference on Wireless Health WH'11, 2011, pp. 8-9.

[23] H. Kim, S. Kim, N. Van Helleputte, A. Artes, M. Konijnenburg, J. Huisken, C. Van Hoof, and R. F. Yazicioglu, "A configurable and lowpower mixed signal SoC for portable ECG monitoring applications," IEEE Trans. Biomed. Circuits Syst., vol. 8, no. 2, pp. 257-267, 2014.

[24] C. J. Deepu, X. Y. Xu, X. D. Zou, L. B. Yao, and Y. Lian, "An ECG-onchip for wearable cardiac monitoring devices," in Proceedings - 5th 
IEEE International Symposium on Electronic Design, Test and Applications, DELTA 2010, 2010, pp. 225-228.

[25] C. J. Deepu, X. Zhang, S. Member, W. Liew, D. Liang, T. Wong, S. Member, and Y. Lian, "An ECG-on-Chip With $535 \mathrm{nW} /$ Channel Integrated Lossless Data Compressor for Wireless Sensors," IEEE J. Solid-State Circuits, vol. 49, no. 11, pp. 2435-2448, 2014.

[26] E. Spanò, S. Di Pascoli, G. Iannaccone, "An Intragrid implementation embedded in an Internet of Things platform", in Proc. 2013 IEEE $18^{\text {th }}$ International Workshop on CAMAD, Germany, pp. 134-138.

[27] E. Spanò, L. Niccolini, S. Di Pascoli, G. Iannaccone, "Last-Meter Smart Grid Embedded in an Internet-of-Things Platform", IEEE Transactions on Smart Grid, vol. 6, no. 1, pp. 468-476, Jan. 2015.

[28] Maxim, "Introduction to electrocardiographs", Tutorial 4693, May 2010.

[29] Texas Instrument, "Analog Front-End Design for ECG Systems Using Delta-Sigma ADCs", Application Report, SBAA160A, March 2009, Revised April 2010.

[30] B.B. Winter, J.G. Webster, "Driven-right-leg circuit design," IEEE Trans. Biomed. Engineering, vol.BME-30, no.1, pp.62-66, Jan. 1983.

[31] E.M. Spinelli, N.H. Martinez, M.A. Mayosky, "A transconductance driven-right-leg circuit," IEEE Transactions on Biomedical Engineering, vol.46, no.12, pp.1466-1470, Dec. 1999.

[32] Texas Instrument, "Improving Common-Mode Rejection Using the Right-Leg Driver Amplifier", Application Report, SBAA188, July 2011.

[33] N. V. Thakor, J. G.Webster, "Ground-free ECG recording with two electrodes," IEEE Transactions on Biomedical Engineering, vol. 27, no. 12, pp. 699-804, Dec. 1980.

[34] E. Richard, A.D.C. Chan, "Design of a gel-less two-electrode ECG monitor," IEEE International Workshop on Medical Measurements and Applications Proceedings (MeMeA), 2010, pp.92,96.

[35] N. Hamza, L. Khriji, R. Tourki, "Interference reduction in ECG signal acquisition: Ground electrode removal (case study)", International Conference on Computer Medical Applications, Jan. 2013, pp. 1-4.

[36] Texas Instrument, "24-Bit Analog-to-Digital Converters for Temperature Sensors", SBAS426G, Aug. 2008, Rev. Oct. 2011. Accessed on 17 Feb. 2016.

[37] Freescale Semiconductor, "MC1322x Advanced ZigBee ${ }^{\mathrm{TM}}$ Compliant Platform-in-Package (PiP) for the 2.4 GHz IEEE® 802.15.4 Standard", Datasheet, Document Number: MC1322x, Rev. 1.3 10/2010. Accessed on $17 \mathrm{Feb} 2016$.

[38] "Recommendations for measurement standards in quantitative electrocardiography. The CSE Working Party”, European heart journal, vol. 6, Issue 10, pp. 815-825, Oct. 1985.

[39] P. Kligfield, et al, "Recommendations for the standardization and interpretation of the electrocardiogram", Journal of the American College of Cardiology, vol. 49, no. 10, pp. 1109-1127, Mar. 2007.

[40] L. Cuiwei, Z. Chongxun, T. Changfeng , "Detection of ECG characteristic points using wavelet transforms", IEEE Transactions on Biomedical Engineering, Vol. 42, Issue: 1, pp. 21-28, Jan. 1995.

[41] V.S. Chouhan, S.S. Mehta, S. S., "Total Removal of Baseline Drift from ECG Signal", International Conference on Computing: Theory and Applications (ICCTA '07), 5-7 March 2007, pp.512-515.

[42] F.A. Afsar, M.S. Riaz, M. Arif, "A Comparison of Baseline Removal Algorithms for Electrocardiogram (ECG) Based Automated Diagnosis of Coronory Heart Disease", 3rd International Conference on Bioinformatics and Biomedical Engineering, June 2009, pp.1,4.

[43] B. Taji, S. Shirmohammadi, V. Groza, and I. Batkin, "Impact of Skin-Electrode Interface on Electrocardiogram Measurements Using Conductive Textile Electrodes," IEEE Transaction on Instrumentation and Measurement, vol. 63, issue 6, pp. 1412 - 1422, June 2014.

[44] C. D. Oster, "Proper Skin Prep Helps Ensure ECG Trace Quality”, 3M Health Care, St. Paul, USA.

[45] Texas Instruments, "Wireless Heart Rate Monitor Reference Design", http://www.ti.com/lit/ug/tidu195a/tidu195a.pdf Accessed 17 Feb. 2016.

[46] L Seung-Chul and C Wan-Young, "A Robust Wearable u-Healthcare Platform in Wireless Sensor Network", Journal of Communication and Networks, vol. 16, no. 4, pp. 465-74, Aug. 2014.

[47] H Gjoreski, A Rashkovska, Si Kozina, M Luštrek, M Gams, "Telehealth using ECG Sensor and Accelerometer", $37^{\text {th }}$ International Convention on Information and Communication Technology, Electronics and Microelectronics, May 2014, pp. 270-4.

[48] K.BregarandV.Avbelj,"Multi-FunctionalWirelessBodySensor Analysis of Autonomy," $36^{\text {th }}$ International Convention on Information and Communication Technology, Electronics and Microelectronics, May 2013, pp. 346-349.
[49] Emil Valchinov, Athanasios Antoniou, Konstantinos Rotas and Nicolas Pallikarakis, "Wearable ECG System for Health and Sports Monitoring", 4th International Conference on Wireless Mobile Communication and Healthcare, nov 2914, Athens, 2014, pp. 63-66.

[50] D.J.C. MacKay, "Information Theory, Inference, and Learning Algorithms", Oxford University Press, pp 628, 2006.

[51] AL Goldberger, LAN Amaral, L Glass, JM Hausdorff, PCh Ivanov, RG Mark, JE Mietus, GB Moody, C-K Peng, HE Stanley. "PhysioBank, PhysioToolkit, and PhysioNet: Components of a New Research Resource for Complex Physiologic Signals," PubMed, Circulation 101(23):e215-e220, Jun 2000.

[52] J. Daemen, V. Rijmen, "The design of Rijndael: AES - the Advanced Encryption Standard", Springer-Verlag, Berlin - New York, 2002.

[53] ZigBee Alliance, "ZigBee Specification", Document 053474r17, Oct. 2007.

[54] ZigBee Alliance, "Home Automation Profile Specification", Document $053520 r 25$, Oct. 2007.

[55] G. Iannaccone, E. Spanò, S. Di Pascoli, unpublished.

[56] R. H. Walden "Analog-to-digital converter survey and analysis", IEEE J. Sel. Areas Commun., vol. 17, no. 4, pp.539 -550, Apr. 1999.

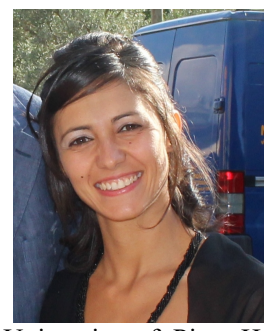

Elisa Spanò (StM'2013) was born in Polistena (RC), Italy, on September 14, 1981. She received the M.S. degree in electronic engineering from the University of Messina, Italy, in 2006, and the Ph.D. degree in Applied Electromagnetism in Electrical and Biomedical Engineering, Electronics, Smart sensors, and Nano-technologies from the University of Pisa, Pisa, Italy, in 2014.

She is currently a Research Fellow with the University of Pisa. Her current research interests include wireless sensor networks (WSN), internet of things (IoT) platforms, and smart grid technologies.

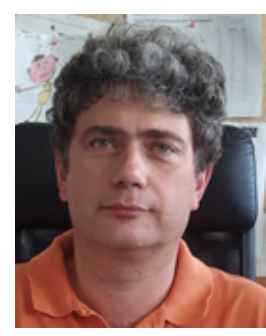

Stefano Di Pascoli was born in Cremona, Italy, in 1967. He received the M.S degree in electronic engineering from the University of Pisa, Pisa, Italy, in 1992, and the Ph.D. degree in electronic engineering from Scuola Superiore S.Anna, Pisa, in 1997.

From 1996 to 2005, he was a Research Assistant and is currently an Associate Professor with the Dipartimento di Ingegneria dell'Informazione, University of Pisa. His current research interests include reliability and electromigration in circuit interconnections, the design of low power radio systems, wireless sensors for home automation and bioengineering applications, and embedded microprocessor applications. He has authored and co-authored over 35 articles.

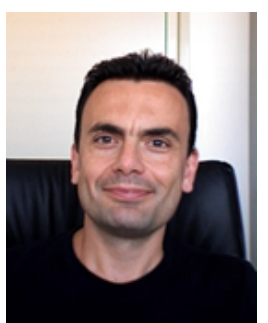

Giuseppe Iannaccone (M'98-SM'10) received the M.S. and Ph.D. degrees in electrical engineering from the University of Pisa, Pisa, Italy, in 1992 and 1996, respectively.

$\mathrm{He}$ is Professor of Electronics with the University of Pisa. His current research interests include nanoelectronics and semiconductor devices, foundations of quantum transport and noise, analog circuit design, and design of smart systems. He has published over 170 papers in peer-reviewed journals and over 130 papers in proceedings of international conferences. He has coordinated a few European and national projects involving multiple partners, and has acted as the Principal Investigator in several research projects funded by public agencies at the European and national levels, and by private organizations. He acts as a reviewer for a few funding agencies in Europe and is or has been in the technical committee of several international conferences in the field of semiconductor technology and design. Prior to joining the University of Pisa in 1996, he was a Researcher with the Italian National Research Council. 